This is the Accepted Manuscript version of an article accepted for publication in the Conference Proceedings of the Society for Experimental Mechanics Series book series.

Schaeffner M., Platz R. (2017) Linear Parameter-Varying (LPV) Buckling Control of an Imperfect Beam-Column Subject to Time-Varying Axial Loads. In: Barthorpe R., Platz R., Lopez I., Moaveni B., Papadimitriou C. (eds) Model Validation and Uncertainty Quantification, Volume 3.

Conference Proceedings of the Society for Experimental Mechanics Series. Springer, Cham. https://doi.org/10.1007/978-3-319-54858-6_11

\title{
Linear Parameter-Varying (LPV) buckling control of an imperfect beam-column subject to time-varying axial loads
}

\author{
Maximilian Schaeffner ${ }^{1}$ and Roland Platz ${ }^{2}$ \\ 1 Technische Universität Darmstadt, System Reliability and Machine Acoustics SzM, \\ Magdalenenstraße 4, 64289 Darmstadt, Germany, schaeffner@szm.tu-darmstadt.de \\ ${ }^{2}$ Fraunhofer Institute for Structural Durability and System Reliability LBF, \\ Bartningstraße 47, 64289 Darmstadt, Germany
}

\begin{abstract}
In this paper, active buckling control of an imperfect slender beam-column with circular cross-section by piezo-elastic supports and Linear Parameter-Varying (LPV) control is investigated experimentally. The beam-column is loaded by a time-varying axial compressive load resulting in a lateral deflection of the beam-column due to imperfections. A finite element model of the beam-column under axial load is designed as an LPV system. A reduced and augmented modal model is used to design a quadratically stable gain scheduled LPV control. The control is implemented in an experimental test setup and the maximum bearable loads of the beam-column are obtained. Two cases are tested: with and without LPV control or, respectively, active and passive configuration. With the proposed active LPV buckling control it is possible to compensate the influence of beam-column imperfections and to compensate uncertainty in mounting and loading that in passive configuration without LPV control may lead to early buckling. Eventually, the maximum bearable axial compressive load is increased above the theoretical critical buckling load.
\end{abstract}

Keywords: LPV control, active buckling control, uncertainty, imperfect beam-column, piezo-elastic supports

\section{INTRODUCTION}

For passive compressively loaded beam-columns, the theory of buckling has been thoroughly investigated for both ideal and imperfect structures, [1]. Imperfect beam-columns exhibit large lateral deformations for axial loads considerably below the theoretical critical buckling load and, therefore, have lower maximum bearable axial loads. A general approach to passively increase the maximum bearable axial load is to change the geometry, e. g. length and cross-section area, or the material so that the beam-column withstands higher loads. This, however, is sometimes not desirable because of given design constraints. In these cases, active buckling control without significant change in the beam-column's geometry and material provides a suitable approach to increase the maximum bearable axial load of a given structure. Active buckling control of slender beam-columns with different boundary conditions has been investigated numerically and experimentally several times, $[2,3,4,5,6,7,8]$. The investigated structures all had rectangular cross-sections, relatively high slenderness ratios $s$, with $300 \leq s \leq 1760$ and low absolute values of the theoretical critical buckling load. Furthermore, the structures were subject to (quasi-) static axial loads and controllers were also static or switched manually. Often, surface bonded piezoelectric patches were applied to beam-columns with rectangular cross-section to induce active bending moments that counteract the deformation, $[2$, $3,4,5,6]$. The active stabilization concept investigated by earlier own studies $[7,8]$ used piezoelectric stack actuators to apply active lateral forces near the base of a fixed-pinned beam-column with rectangular cross-section.

To the authors' knowledge, active buckling control of beam-columns with circular cross-section has not yet been investigated except in own works, [9, 10]. In both investigations, active buckling control of a circular beam-column with relatively low slenderness ratio $s=108$ with piezo-elastic supports at both ends and stabilized by integral linear quadratic control (LQR) was investigated numerically and experimentally for (quasi-)static axial loads. The integral LQR was able to compensate initial deformation that could lead to buckling and increase the maximum bearable 
load of the beam-column in numerical simulation and experiment. Control matrices were switched manually due to increasing axial loads and the maximum bearable axial load was limited by the theoretical critical buckling load of the beam-column with piezo-elastic supports at both ends. Now, in this paper, active buckling control of the same slender beam-column with improved piezo-elastic supports and LPV control is investigated experimentally. The beam-column is loaded by a time-varying axial compressive load resulting in a lateral deflection of the beam-column due to imperfections. The quadratically stable gain scheduled LPV control is designed and implemented in an experimental test setup to account for the axial load dependency of the beam-columns lateral stiffness.

\section{SYSTEM DESCRIPTION AND MATHEMATICAL MODEL OF BEAM-COLUMN SYSTEM}

In the following, first the investigated beam-column with piezo-elastic supports and the experimental test setup for active LPV buckling control are presented. Second, a mathematical finite element (FE) model of the axially loaded beam-column is derived and validated with experimental results. The investigated system is a slender beam-column made of aluminum alloy EN AW-7075 with length $l_{\mathrm{b}}=400 \mathrm{~mm}$ and circular solid cross-section with radius $r_{\mathrm{b}}=4 \mathrm{~mm}$, figure 1. It has a Young's modulus $E_{\mathrm{b}}=75.8 \cdot 10^{3} \mathrm{~N} / \mathrm{mm}^{2}$ and density $\varrho_{\mathrm{b}}=2.79 \cdot 10^{-3} \mathrm{~g} / \mathrm{mm}^{3}$, all assumed to be constant across the entire beam-column length.

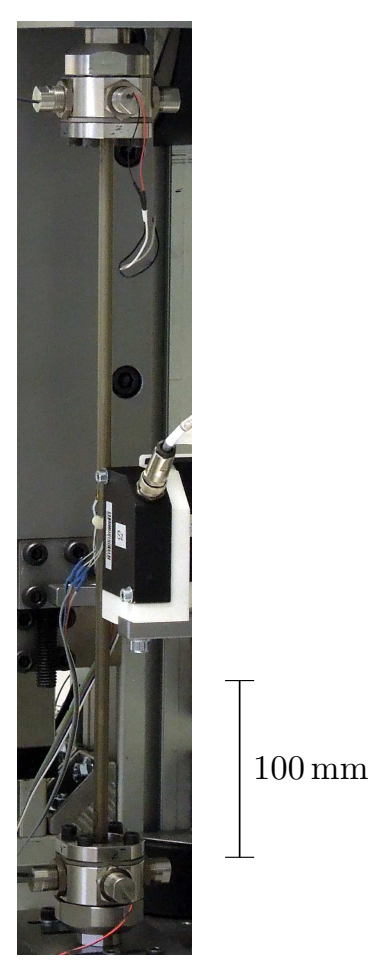

(a)

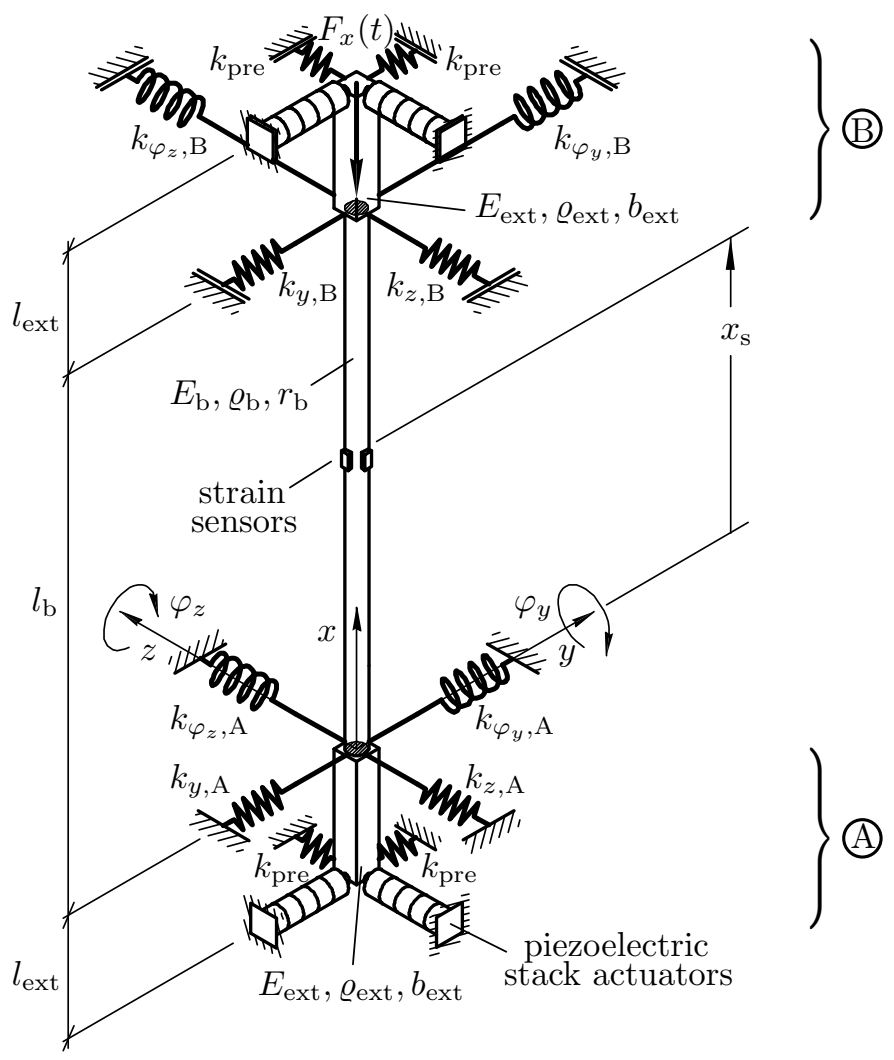

(b)

Fig. 1 Beam-column system, (a) beam-column with piezo-elastic supports for experimental test, (b) sketch of beam-column

The beam-column is embedded in two piezo-elastic supports $\mathrm{A}$ at $x=0$ and $\mathrm{B}$ at $x=l_{\mathrm{b}}$. At support $\mathrm{B}$, a time-varying axial load $F_{x}(t)$ is applied. Strain gauges at sensor position $x_{\mathrm{s}}=l_{\mathrm{b}} / 2$ are used to measure the surface strain of the beam-column in $y$ - and $z$-direction. Figure 2 shows sectional views of piezo-elastic support A, [11]. The central element of the support are two concave-shaped elastic membrane springs made of spring steel 1.1248 that bear the axial compressive and lateral loads and allow rotations in any plane perpendicular to the $x$-axis, figures $2 \mathrm{a}$ and 2b. They are represented by rotational stiffness $k_{\varphi_{y}, \mathrm{~A}}=k_{\varphi_{z}, \mathrm{~A}}=k_{\varphi_{y}, \mathrm{~B}}=k_{\varphi_{z}, \mathrm{~B}}=k_{\mathrm{r}}$ and lateral stiffness $k_{y, \mathrm{~A}}=k_{z, \mathrm{~A}}=k_{y, \mathrm{~B}}=k_{z, \mathrm{~B}}=k_{1}$ that are the same for both supports $\mathrm{A}$ and $\mathrm{B}$ and in $y$ - and $z$-direction, figure $1 \mathrm{~b}$. 


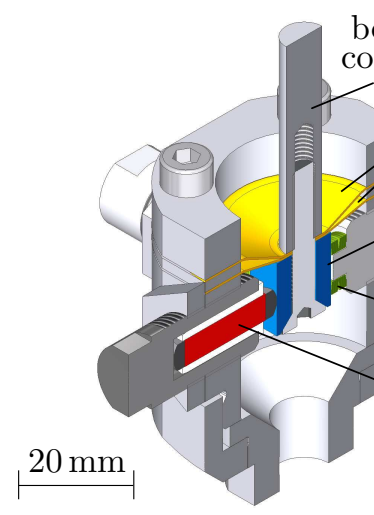

(a)

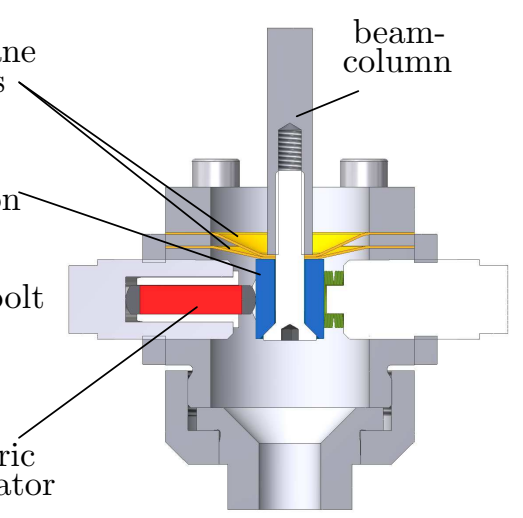

(b)

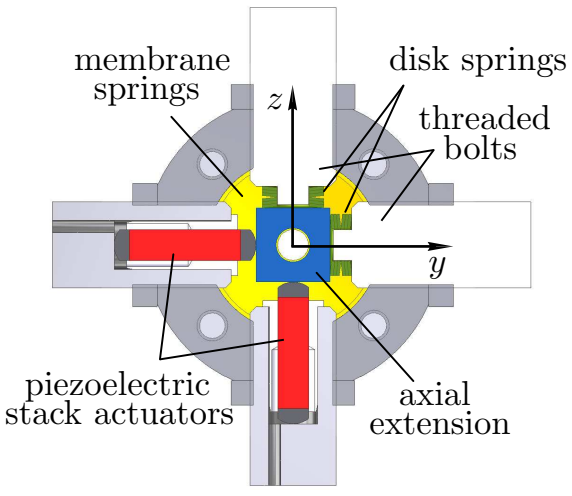

(c)

Fig. 2 Sectional views of piezo-elastic support A, (a) 3D section through $x$-y-plane, (b) 2D section through $x$-y-plane, (c) $2 \mathrm{D}$ section bottom view through actuator-plane

In each piezo-elastic support A and B at $x=-l_{\text {ext }}$ and $x=l_{\mathrm{b}}+l_{\text {ext }}$, two piezoelectric stack actuators Piezomechanik PST 150 are arranged orthogonal to each other and orthogonal to the beam-column's $x$-axis, acting in $y$ - and $z$-direction, figure 2c. Each actuator is mechanically prestressed by an allocated stack of disc springs with stiffness $k_{\text {pre }}=2.6 \cdot 10^{3} \mathrm{~N} / \mathrm{mm}$. The initial deflection of the disk springs is adjusted by threaded bolts. The piezoelectric stack actuators exert lateral forces to the beam-column axial extension with quadratic cross-section and relatively high bending stiffness made of hardened steel 1.4301 with length $l_{\text {ext }}=7.9 \mathrm{~mm}$, edge length $b_{\text {ext }}=6 \mathrm{~mm}$, Young's modulus $E_{\text {ext }}=210.0 \cdot 10^{3} \mathrm{~N} / \mathrm{mm}^{2}$ and density $\varrho_{\text {ext }}=7.81 \cdot 10^{-3} \mathrm{~g} / \mathrm{mm}^{3}$. The extension works as cantilever beam end beyond the elastic membrane springs for each support A and B. This way, controlled active lateral forces by the two piezoelectric stack actuators act in arbitrary directions orthogonal to the beam-column's longitudinal $x$-axis. They result in bending moments acting in arbitrary directions at the beam-column ends in both piezo-elastic supports A and B.

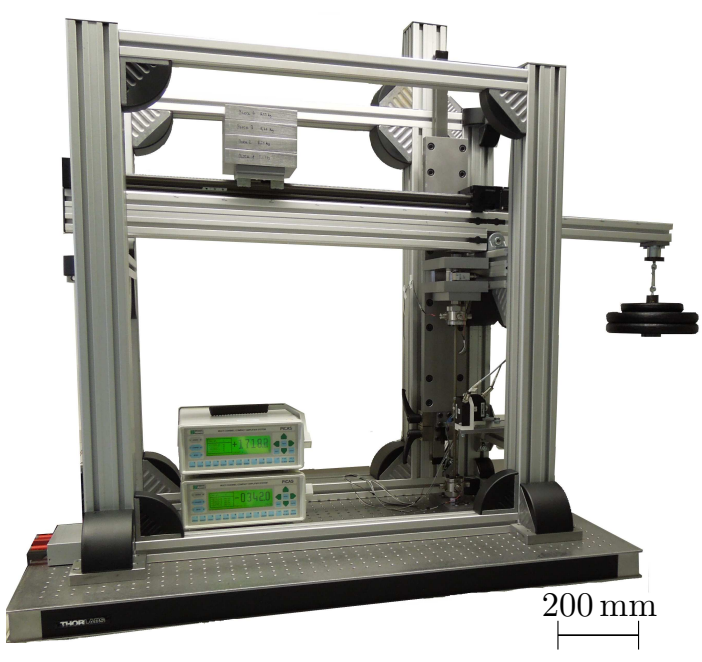

(a)

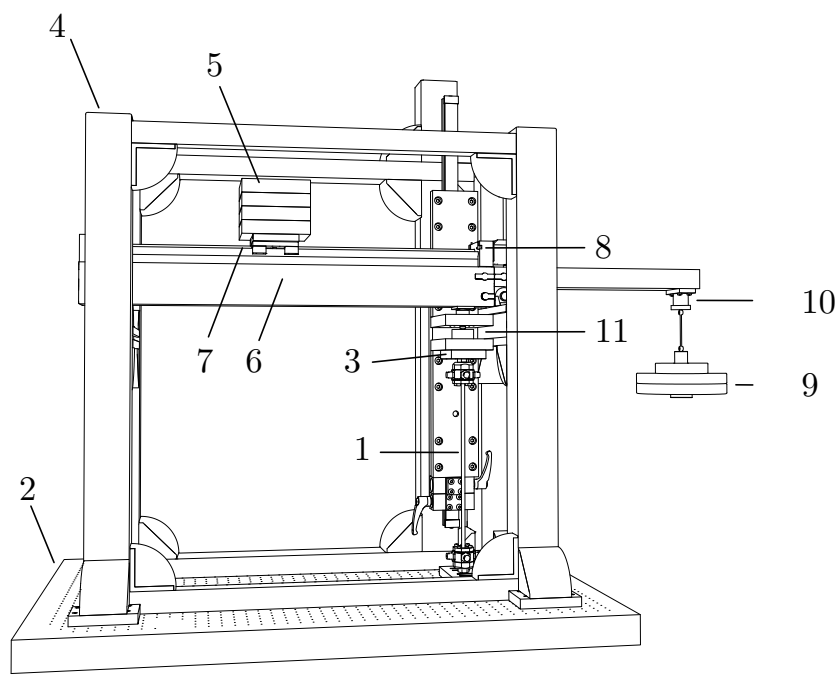

(b)

Fig. 3 Experimental test setup for active buckling control, (a) photo of test setup, (b) CAD sketch of test setup (without strain gauge amplifiers)

For experimental investigations, the beam-column system is integrated in an experimental test setup. Figure 3 shows a photo and a CAD sketch of the experimental test setup for the active buckling control of the slender beam-column 
with circular cross-section and piezo-elastic supports, 1, figure 1a. The lower piezo-elastic support A is fixed on a baseplate 2, the upper piezo-elastic support B is fixed to a parallel guidance 3 that is connected to a stiff frame structure 4. The beam-column is loaded via a high lever ratio by a mass 5 of up to $30 \mathrm{~kg}$ that is placed on a hinged beam 6 . The lever ratio and, therefore, the axial load are increased by shifting the mass in nearly horizontal plane via a linear axle 7, operated by a stepper motor 8 . An additional optional weight 9 of up to $15 \mathrm{~kg}$ reduces the axial load on the beam-column. It can be released by an electromagnet 10, thus generating a step axial compressive load. The axial load is measured by a load cell 11. With the presented test setup, ramp-shaped axial loads between the static load $F_{\min }=330 \mathrm{~N}$ of the parallel guidance 3 , and $F_{\max } \approx 5000 \mathrm{~N}$ as well as step-shaped axial loads of up to $F_{\text {step }}=800 \mathrm{~N}$ can be generated. This is sufficient to generate static and time-dependent axial loads $F_{x}(t)$ considerably above the theoretical EULER buckling load of the fixed-fixed beam-column with $F_{x, \mathrm{E}}=4 \pi^{2} E_{\mathrm{b}} I_{\mathrm{b}} / l_{\mathrm{b}}^{2}=3760 \mathrm{~N}$, [1].

\subsection{FINITE ELEMENT STATE SPACE MODEL OF BEAM-COLUMN SYSTEM}

To describe the lateral vibration of the axially loaded beam-column in state space, a finite element (FE) model is the basis. The beam-column and the stiff axial extensions are modeled by the FE equation of motion

$$
\boldsymbol{M} \ddot{\boldsymbol{r}}+\boldsymbol{D} \dot{\boldsymbol{r}}+\left(\boldsymbol{K}_{\mathrm{e}}-F_{x}(t) \boldsymbol{K}_{\mathrm{g}}\right) \boldsymbol{r}=\boldsymbol{B}_{0} \boldsymbol{u}
$$

and output equation

$$
\boldsymbol{y}=\left[\begin{array}{c}
\varepsilon_{\mathrm{s}, y} \\
\varepsilon_{\mathrm{s}, z}
\end{array}\right]=\boldsymbol{C}_{0} \boldsymbol{r} .
$$

In (1), $\boldsymbol{r}$ is the $[4 N \times 1] \mathrm{FE}$ displacement vector for $N$ nodes and $4 N$ degrees of freedom with two translational and two rotational displacements in and around $y$ - and $z$-direction. $\boldsymbol{M}$ is the mass matrix, $\boldsymbol{D}$ is the damping matrix, $\boldsymbol{K}_{\mathrm{e}}$ is the elastic stiffness matrix and $\boldsymbol{K}_{\mathrm{g}}$ is the geometric stiffness matrix that describes the influence of axial load $F_{x}(t)$ on the beam-column's lateral stiffness, all $[4 N \times 4 N]$. The stiffness of the elastic membrane springs, disk springs and piezoelectric stack actuators are included in the stiffness matrices.

The right side of (1) represents the active control forces acting simultaneously at both supports A and B that are summarized in control input vector

$$
\boldsymbol{u}=\left[\begin{array}{c}
F_{\mathrm{a}, y} \\
F_{\mathrm{a}, z}
\end{array}\right]
$$

and that are derived in section 3.2. The $[4 N \times 2]$ control input matrix

$$
\boldsymbol{B}_{0}=\left[\begin{array}{lllllllll}
1 & 0 & 0 & 0 & \cdots & 1 & 0 & 0 & 0 \\
0 & 1 & 0 & 0 & \cdots & 0 & 1 & 0 & 0
\end{array}\right]^{T}
$$

allocates the active forces of the piezoelectric stack actuators to the lateral degrees of freedom of the first and last nodes of the FE model. In (2), the $[2 \times 4 N]$ output matrix $\boldsymbol{C}_{0}$ allocates the surface strains $\boldsymbol{y}$ to the FE displacement vector $\boldsymbol{r},[9]$. For convenience, (1) and (2) are written in state space representation

$$
\begin{aligned}
\dot{\boldsymbol{x}}_{\mathrm{FE}} & =\underbrace{\left(\left[\begin{array}{cc}
\mathbf{0} & \mathbf{I} \\
-\boldsymbol{M}^{-1} \boldsymbol{K}_{\mathrm{e}} & -\boldsymbol{D}
\end{array}\right]+F_{x}(t)\left[\begin{array}{cc}
\mathbf{0} & \mathbf{0} \\
\boldsymbol{M}^{-1} \boldsymbol{K}_{\mathrm{g}} & \mathbf{0}
\end{array}\right]\right)}_{[8 N \times 8 N]} \boldsymbol{x}_{\mathrm{FE}}+\underbrace{\left[\begin{array}{c}
\mathbf{0} \\
\boldsymbol{M}^{-1} \boldsymbol{B}_{0}
\end{array}\right]}_{[8 N \times 2]} \boldsymbol{u} \\
\boldsymbol{y} & =\underbrace{\left[\begin{array}{ll}
\boldsymbol{C}_{0} & \mathbf{0}
\end{array}\right]}_{[2 \times 8 N]} \boldsymbol{x}_{\mathrm{FE}},
\end{aligned}
$$

with $[8 N \times 1]$ FE state vector $\boldsymbol{x}_{\mathrm{FE}}=[\boldsymbol{r}, \dot{\boldsymbol{r}}]^{T}$ and zero and identity matrices $\mathbf{0}$ and $\mathbf{I}$ of appropriate dimensions. The full FE state space model of the beam-column as short form of (5) can be written as

$$
\begin{aligned}
\dot{\boldsymbol{x}}_{\mathrm{FE}} & =\boldsymbol{A}_{\mathrm{FE}}\left(F_{x}(t)\right) \boldsymbol{x}_{\mathrm{FE}}+\boldsymbol{B}_{\mathrm{FE}} \boldsymbol{u} \\
\boldsymbol{y} & =\boldsymbol{C}_{\mathrm{FE}} \boldsymbol{x}_{\mathrm{FE}},
\end{aligned}
$$

[9]. The influence of axial load $F_{x}(t)$ on the system behavior is described by system matrix $\boldsymbol{A}_{\mathrm{FE}}\left(F_{x}(t)\right)$. In the following, the time-dependent axial force will be written as $F_{x}=F_{x}(t)$. 


\subsection{SYSTEM IDENTIFICATION AND MODEL VALIDATION OF BEAM-COLUMN SYSTEM}

To validate the FE beam-column model, the transfer behavior of model (6) and experiment are compared. The two frequency response functions

$$
H_{y}\left(F_{x}, \Omega\right)=\frac{\varepsilon_{\mathrm{s}, y}(\Omega)}{F_{\mathrm{a}, y}(\Omega)} \quad \text { and } \quad H_{z}\left(F_{x}, \Omega\right)=\frac{\varepsilon_{\mathrm{s}, z}(\Omega)}{F_{\mathrm{a}, z}(\Omega)}
$$

represent the surface strain responses $\varepsilon_{\mathrm{s}, y / z}$ at $x=x_{\mathrm{s}}$ in $y$ - and $z$-direction, figure $1 \mathrm{~b}$, to an excitation by the active forces $F_{\mathrm{a}, y / z}$ at $x=-l_{\text {ext }}$ and $x=l_{\mathrm{b}}+l_{\text {ext }}$ in $y$ - and $z$-direction with excitation angular frequency $\Omega$. Figure 4a shows the amplitude $\left|H_{y / z}\right|$ and phase $\arg H_{y / z}$ of the frequency response functions $H_{y}$ and $H_{z}$ for the beam-column loaded with static axial load $F_{x}=2000 \mathrm{~N}$ for model and experiment. Amplitude and phase of the frequency response functions from model (7) and both $y$ - and $z$-direction in experiment coincide well for considered frequency range $0 \mathrm{~Hz}<\Omega / 2 \pi<250 \mathrm{~Hz}$. The first resonance frequency $\omega_{1} / 2 \pi=142 \mathrm{~Hz}$ at the maximum amplitude $\max \left(\left|H_{y / z}\right|\right)=\left|H_{y / z}\left(F_{x}, \Omega=\omega_{1}\right)\right|$ also matches for numerical simulation and experiment.

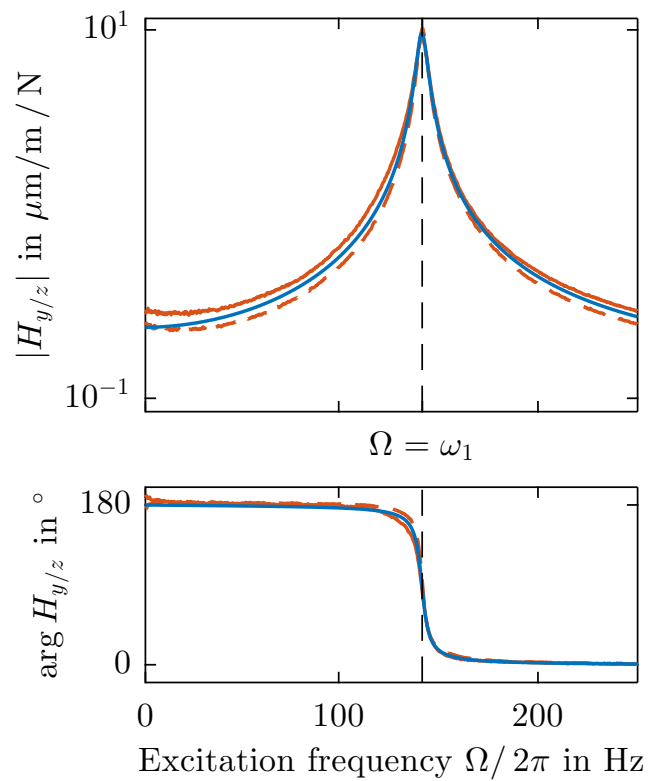

(a)

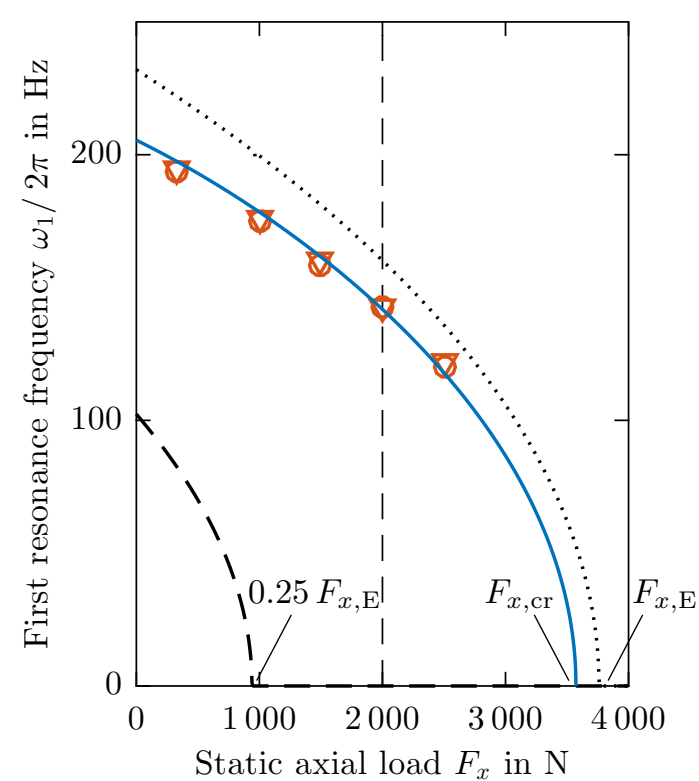

(b)

Fig. 4 System identification of beam-column system, (a) amplitude $\left|H_{y / z}\right|$ and phase $\arg H_{y / z}$ of frequency response functions $H_{y / z}$ for static axial load $F_{x}=2000 \mathrm{~N}$ from numerical simulation $(-)$ and experiment in $y$-direction (-) and $z$-direction (- ), (b) first resonance frequency $\omega_{1} / 2 \pi$ for varying axial load $0 \mathrm{~N}<F_{x}<4000 \mathrm{~N}$ from simulation $(-)$ and experiments in $y$-direction $(\nabla)$ and $z$-direction $(\bigcirc)$ as well as ideal pinned-pinned $(--)$ and fixed-fixed ( ... ) beam-column

With increasing static axial load $F_{x}$, the first resonance frequency of the beam-column decreases. Figure $4 \mathrm{~b}$ shows the change of the first resonance frequency $\omega_{1}\left(F_{x}\right) / 2 \pi$ due to increasing static axial load of the experimental beam-column at five designated axial loads $F_{x}=330 \mathrm{~N}, 1000 \mathrm{~N}, 1500 \mathrm{~N}, 2000 \mathrm{~N}, 2500 \mathrm{~N}$ and for the numerical simulation from $0 \mathrm{~N}<F_{x}<4000 \mathrm{~N}$ for the beam-column with piezo-elastic supports and for ideal pinned-pinned and fixed-fixed supports. In the experiment, the system identification can only be performed up to axial loads of $F_{x}=2500 \mathrm{~N}$ due to the high static deformations of the uncontrolled beam-column. In the numerical simulation, the first resonance frequency $\omega_{1}\left(F_{x}\right) / 2 \pi$ is calculated by solution of the well-known eigenvalue problem

$$
\operatorname{det}\left[\left(\boldsymbol{K}_{\mathrm{e}}-F_{x} \boldsymbol{K}_{\mathrm{g}}\right)-\omega_{1}^{2} \boldsymbol{M}\right]=0 .
$$

As seen in figure $4 \mathrm{~b}$, the first resonance frequency decreases with increasing axial load $F_{x}$ and the FE model (6) and the experiment agree well for the axial load range of $330 \mathrm{~N}<F_{x}<2500 \mathrm{~N}$. In the numerical simulation, the first 
resonance frequency reaches zero for the critical buckling load that can, therefore, be calculated from the elastic and geometric stiffness matrices by solution of the modified eigenvalue problem (8) for $\omega_{1}=0$ by

$$
\operatorname{det}\left[\boldsymbol{K}_{\mathrm{e}}-F_{x} \boldsymbol{K}_{\mathrm{g}}\right]=0,
$$

[12]. The decreasing course of the first resonance frequency $\omega_{1} / 2 \pi\left(F_{x}\right)$ for the beam-column with piezo-elastic supports in figure $4 \mathrm{~b}$ is near the decreasing course of the first resonance frequency of the beam-column ideally fixed at supports A and B, whereas the distance between both curves decreases with increasing axial load. The slower decrease of the first resonance frequency with increasing axial load $F_{x}$ results from a stiffening effect of the elastic membrane spring's lateral and rotational stiffness. Consequently, the lateral and rotational stiffness are modeled as

$$
k_{1}=k_{\mathrm{l}, \mathrm{e}}+F_{x} k_{\mathrm{l}, \mathrm{g}} \quad \text { and } \quad k_{\mathrm{r}}=k_{\mathrm{r}, \mathrm{e}}+F_{x} k_{\mathrm{r}, \mathrm{g}}
$$

with constant stiffness for the unloaded beam-column $k_{1, \mathrm{e}}=70.5 \cdot 10^{3} \mathrm{~N} / \mathrm{mm}$ and $k_{\mathrm{r}, \mathrm{e}}=388.8 \cdot 10^{3} \mathrm{Nmm} / \mathrm{rad}$ as well as axial force proportional component $k_{\mathrm{l}, \mathrm{g}}=9.5 \cdot 10^{6} \mathrm{~N} / \mathrm{mm}$ and $k_{\mathrm{r}, \mathrm{g}}=480.0 \mathrm{Nmm} / \mathrm{rad}$. The lateral and rotational stiffness are added to the entries of the lateral and rotational degrees of freedom of nodes $n=2$ and $n=N-1$ of the elastic and geometric stiffness matrices $\boldsymbol{K}_{\mathrm{e}}$ and $\boldsymbol{K}_{\mathrm{g}}$. For the given boundary conditions, the critical buckling load is determined to $F_{x, \mathrm{cr}}=3573 \mathrm{~N}$ which is close to the theoretical critical buckling load of the fixed-fixed EULER beam-column $F_{x, \mathrm{E}}=3760 \mathrm{~N}$, figure $4 \mathrm{~b}$.

\section{REDUCED STATE SPACE CONTROL MODEL AND LPV CONTROL}

In the following, first, the FE model of the axially loaded beam-column (6) will be reduced to a modal control model in order to reduce complexity and design the LPV control. The modal beam-column model is augmented to include integrated states to compensate imperfections of the beam-column. Second, the LPV control for active buckling control is derived.

\subsection{MODAL STATE SPACE CONTROL MODEL}

For the full state FE model (1), a number of $N=35$ nodes resulting in $4 N=140$ degrees of freedom and modes is chosen to properly describe the maximum surface strains at sensor position $x_{\mathrm{s}}$ according to (2) and for adequate model validation in figure 4. For controller design, however, the FE model is reduced by modal truncation to only include the first lateral vibration mode for both $y$ - and $z$-direction. Thus, the controller complexity is reduced considerably and the resulting modal beam-column model is observable and controllable with the given sensors and actuators, section 2. Consequently, the FE displacement vector

$$
\boldsymbol{r} \approx \boldsymbol{\Phi} \boldsymbol{q}=\left[\widehat{\boldsymbol{r}}_{1, y}, \widehat{\boldsymbol{r}}_{1, z}\right]\left[\begin{array}{l}
q_{1, y} \\
q_{1, z}
\end{array}\right]
$$

is approximated by modal displacements $\boldsymbol{q}$ via the transformation with the [ $4 N \times 2]$ modal matrix $\boldsymbol{\Phi}$ that includes the first [ $4 N \times 1$ ] eigenvectors $\widehat{\boldsymbol{r}}_{1, y}$ and $\widehat{\boldsymbol{r}}_{1, z}$ in $y$ - and $z$-direction of the FE Model, [13]. Modal matrix $\boldsymbol{\Phi}$ is normalized with respect to mass matrix $\boldsymbol{M}$ leading to the modal mass matrix $\boldsymbol{M}_{\mathrm{m}}$, modal elastic stiffness matrix $\boldsymbol{K}_{\mathrm{m}, \mathrm{e}}$, modal geometric stiffness matrix $\boldsymbol{K}_{\mathrm{m}, \mathrm{g}}$ and modal damping matrix $\boldsymbol{D}_{\mathrm{m}}$

$$
\boldsymbol{M}_{\mathrm{m}}=\boldsymbol{\Phi}^{T} \boldsymbol{M} \boldsymbol{\Phi}=\mathbf{I}, \quad \boldsymbol{K}_{\mathrm{m}, \mathrm{e}}=\boldsymbol{\Phi}^{T} \boldsymbol{K}_{\mathrm{e}} \boldsymbol{\Phi}, \quad \boldsymbol{K}_{\mathrm{m}, \mathrm{g}}=\boldsymbol{\Phi}^{T} \boldsymbol{K}_{\mathrm{g}} \boldsymbol{\Phi} \quad \text { and } \quad \boldsymbol{D}_{\mathrm{m}}=\boldsymbol{\Phi}^{T} \boldsymbol{D} \boldsymbol{\Phi}
$$

with identity matrix $\mathbf{I}$, all $[2 \times 2]$. Using the $[4 \times 1]$ modal state vector with the modal displacements and velocities $\boldsymbol{x}_{\mathrm{m}}=[\boldsymbol{q}, \dot{\boldsymbol{q}}]^{T}$, the modal state space equations of first order are

$$
\begin{aligned}
\dot{\boldsymbol{x}}_{\mathrm{m}} & =\underbrace{\left(\left[\begin{array}{cc}
\mathbf{0} & \mathbf{I} \\
-\boldsymbol{K}_{\mathrm{m}, \mathrm{e}} & -\boldsymbol{D}_{\mathrm{m}}
\end{array}\right]+F_{x}\left[\begin{array}{cc}
\mathbf{0} & \mathbf{0} \\
\boldsymbol{K}_{\mathrm{m}, \mathrm{g}} & \mathbf{0}
\end{array}\right]\right)}_{[4 \times 4]} \boldsymbol{x}_{\mathrm{m}}+\underbrace{\left[\begin{array}{c}
\mathbf{0} \\
\boldsymbol{\Phi}^{T} \boldsymbol{B}_{0}
\end{array}\right]}_{[4 \times 2]} \boldsymbol{u} \\
\boldsymbol{y} & =\underbrace{\left[\begin{array}{ll}
\boldsymbol{C}_{0} \boldsymbol{\Phi} & \mathbf{0}
\end{array}\right]}_{[2 \times 4]} \boldsymbol{x}_{\mathrm{m}},
\end{aligned}
$$


[13]. Equation (13) can also be written in short form

$$
\begin{aligned}
\dot{\boldsymbol{x}}_{\mathrm{m}} & =\boldsymbol{A}_{\mathrm{m}}\left(F_{x}\right) \boldsymbol{x}_{\mathrm{m}}+\boldsymbol{B}_{\mathrm{m}} \boldsymbol{u} \\
\boldsymbol{y} & =\boldsymbol{C}_{\mathrm{m}} \boldsymbol{x}_{\mathrm{m}} .
\end{aligned}
$$

Due to imperfections such as predeformation, eccentric loading or clamping moments that are present in a real beam-column system, the controller needs to have an additional integral term to avoid a static controller error. Therefore, the modal state vector $\boldsymbol{x}_{\mathrm{m}}$ is augmented by the integral of the modal displacements

$$
\boldsymbol{x}_{\mathrm{int}}=\int_{t_{\mathrm{LPV}}}^{\infty} \boldsymbol{q} \mathrm{d} t
$$

starting from $t_{\mathrm{LPV}}$ to get the new $[6 \times 1]$ state vector $\boldsymbol{x}=\left[\boldsymbol{x}_{\mathrm{m}}, \boldsymbol{x}_{\mathrm{int}}\right]^{T}$. With the first derivative of the new state vector

$$
\dot{\boldsymbol{x}}_{\mathrm{int}}=\boldsymbol{q}=\left[\begin{array}{ll}
\mathbf{I} & \mathbf{0}
\end{array}\right] \boldsymbol{x}_{\mathrm{m}},
$$

the augmented state space system including the integral term can be written as

$$
\begin{aligned}
& \dot{\boldsymbol{x}}=\underbrace{\left[\begin{array}{cc}
\boldsymbol{A}_{\mathrm{m}}\left(F_{x}\right) & \mathbf{0} \\
{\left[\begin{array}{ll}
\mathbf{I} & \mathbf{0}
\end{array}\right]} & \mathbf{0}
\end{array}\right]}_{[6 \times 6]} \boldsymbol{x}+\underbrace{\left[\begin{array}{c}
\boldsymbol{B}_{\mathrm{m}} \\
\mathbf{0}
\end{array}\right]}_{[6 \times 2]} \boldsymbol{u} \\
& \boldsymbol{y}=\underbrace{\left[\begin{array}{cc}
\boldsymbol{C}_{\mathrm{m}} & \mathbf{0}
\end{array}\right]}_{[2 \times 6]} \boldsymbol{x},
\end{aligned}
$$

[14]. In short form, the final state space system (17) of the beam-column system in figure 1 is written as

$$
\begin{aligned}
& \dot{\boldsymbol{x}}=\boldsymbol{A}\left(F_{x}\right) \boldsymbol{x}+\boldsymbol{B} \boldsymbol{u} \\
& \boldsymbol{y}=\boldsymbol{C} \boldsymbol{x} .
\end{aligned}
$$

\subsection{QUADRATICALLY STABLE GAIN-SCHEDULED LPV CONTROL}

The FE state space model (6) as well as the final controller state space model (18) are LPV systems in which system matrices $\boldsymbol{A}_{\mathrm{FE}}\left(F_{x}\right)$ and $\boldsymbol{A}\left(F_{x}\right)$ depend on axial load $F_{x}$. There are different control approaches to deal with the parameter-dependency of LPV systems. One approach is to use robust control in which a single controller is used for all occurring axial load variations with respect to amplitude and time-dependency. Due to the large variation of axial loads and the transition from sub- to supercritical axial loads, robust control is not favorable and has not been used so far. The approach pursued in earlier own studies $[7,8,9,10]$ calculated static control matrices for a number of different axial loads that were manually switched, resulting in discontinuities in control input (3).

Now, in this investigation, active buckling control of the circular beam-column is achieved by a quadratically stable gain-scheduled LPV control. In this approach, a continuous control input according to (3) with

$$
\boldsymbol{u}=\boldsymbol{K}_{\mathrm{LPV}}\left(F_{x}\right) \boldsymbol{x},
$$

is achieved by $[2 \times 6]$ control matrix $\boldsymbol{K}_{\mathrm{LPV}}\left(F_{x}\right)$ as a linear function of the axial load $F_{x}$ with

$$
\boldsymbol{K}_{\mathrm{LPV}}\left(F_{x}\right)=\frac{F_{x, 2}-F_{x}}{F_{x, 2}-F_{x, 1}} \boldsymbol{K}_{1}+\frac{F_{x}-F_{x, 1}}{F_{x, 2}-F_{x, 1}} \boldsymbol{K}_{2} .
$$

In (20) $F_{x, 1}=300 \mathrm{~N}$ and $F_{x, 2}=4000 \mathrm{~N}$ are the minimum and maximum considered axial loads for the controller that define the vertices of the LPV system $\boldsymbol{A}_{1}=\boldsymbol{A}\left(F_{x, 1}\right)$ and $\boldsymbol{A}_{2}=\boldsymbol{A}\left(F_{x, 2}\right)$ and $\boldsymbol{K}_{1}$ and $\boldsymbol{K}_{2}$ are the control matrices calculated for the respective systems. The resulting controller stabilizes the beam-column for the entire range of considered axial loads, [15].

In order to weight the different states and control inputs, an additional $[8 \times 1]$ performance variable

$$
\boldsymbol{z}=\boldsymbol{C}_{z}\left(F_{x}\right) \boldsymbol{x}+\boldsymbol{D}_{z} \boldsymbol{u},
$$


is introduced. The $[8 \times 6]$ matrix $\boldsymbol{C}_{z}\left(F_{x}\right)$ includes weights on system state vector $\boldsymbol{x}$ and the $[8 \times 2]$ matrix $\boldsymbol{D}_{z}$ includes weights on control input $\boldsymbol{u}$. For the experimental active buckling control, the performance weight matrices in (21) are chosen as

$$
\boldsymbol{C}_{z, 1}=\left[\begin{array}{cccccc}
5 & 0 & 0 & 0 & 0 & 0 \\
0 & 5 & 0 & 0 & 0 & 0 \\
0 & 0 & 1 & 0 & 0 & 0 \\
0 & 0 & 0 & 1 & 0 & 0 \\
0 & 0 & 0 & 0 & 10 & 0 \\
0 & 0 & 0 & 0 & 0 & 10 \\
0 & 0 & 0 & 0 & 0 & 0 \\
0 & 0 & 0 & 0 & 0 & 0
\end{array}\right], \boldsymbol{C}_{z, 2}=\left[\begin{array}{cccccc}
50 & 0 & 0 & 0 & 0 & 0 \\
0 & 50 & 0 & 0 & 0 & 0 \\
0 & 0 & 10 & 0 & 0 & 0 \\
0 & 0 & 0 & 10 & 0 & 0 \\
0 & 0 & 0 & 0 & 1 & 0 \\
0 & 0 & 0 & 0 & 0 & 1 \\
0 & 0 & 0 & 0 & 0 & 0 \\
0 & 0 & 0 & 0 & 0 & 0
\end{array}\right] \text { and } \boldsymbol{D}_{z}=\left[\begin{array}{cc}
0 & 0 \\
0 & 0 \\
0 & 0 \\
0 & 0 \\
0 & 0 \\
0 & 0 \\
30 & 0 \\
0 & 30
\end{array}\right] .
$$

Equation (18) and (21) define the LPV system for the controller. Consequently, the axial load dependent system matrix and state performance matrix are $\boldsymbol{A}_{i}=\boldsymbol{A}\left(F_{x, i}\right)$ and $\boldsymbol{C}_{z, i}=\boldsymbol{C}_{z}\left(F_{x, i}\right)$ for $i=1,2$.

The control law leads to the control input $\boldsymbol{u}(3)$ and (19) so that the expected value of the quadratic performance index

$$
J=\lim _{t \rightarrow \infty} \mathbb{E}\left[\boldsymbol{z}^{T} \boldsymbol{z}\right]<\mu
$$

is minimized, [15]. The control for matrices $\boldsymbol{K}_{i}=\boldsymbol{K}\left(F_{x, i}\right)$ for $i=1,2$ in (20) are calculated by

$$
\boldsymbol{K}_{i}=\boldsymbol{L}_{i} \boldsymbol{X}^{-1}
$$

In (24), $\boldsymbol{L}_{i}$ and $\boldsymbol{X}$ are the solution of linear matrix inequalities (LMI)

$$
\left.\begin{array}{rl}
\boldsymbol{A}_{i} \boldsymbol{X}+\boldsymbol{X} \boldsymbol{A}_{i}^{T}+\boldsymbol{B} \boldsymbol{L}_{i}+\boldsymbol{L}_{i}^{T} \boldsymbol{B}^{T} & \prec 0 \\
\boldsymbol{Z}_{i} & \boldsymbol{C}_{z, i} \boldsymbol{X}+\boldsymbol{D}_{z} \boldsymbol{L}_{i} \\
\boldsymbol{X} \boldsymbol{C}_{z, i}^{T}+\boldsymbol{L}_{i}^{T} \boldsymbol{D}_{z}^{T} & \boldsymbol{X}
\end{array}\right] \succ 0
$$

in which the left hand sides of have to be negative and positive definite, respectively. The LMI's are solved by $Y A L M I P,[16]$, for all $i=1,2$ to minimize

$$
\operatorname{trace}\left(\boldsymbol{Z}_{i}\right)<\mu \text {. }
$$

The resulting control input (19) provides the minimum performance variable (21) for the chosen weights (22).

\section{EXPERIMENTAL RESULTS FOR ACTIVE BUCKLING CONTROL}

Experimental studies to validate the LPV control (19) are conducted on the experimental test setup for active buckling control, figure 3 . The axial load $F_{x}$ is measured by load cell 11 in figure $3 \mathrm{~b}$ as input for the control matrix $\boldsymbol{K}_{\mathrm{LPV}}\left(F_{x}\right)$ in (20). For that, the surface strains according to (2) are measured with strain gauge sensors on the beam-column, figure 1, that are transformed into modal displacements of the beam-column in $y$ - and $z$-direction. The amplified signal which is filtered by analogue elliptic lowpass anti-aliasing filters is fed into a real-time $d S P A C E 1103$ system. The deformation of the beam-column in $y$ - and $z$-direction is approximated by the modal displacements $\boldsymbol{q}$ that, due to the invertibility of the $[2 \times 2]$ matrix $\left(\boldsymbol{C}_{0} \boldsymbol{\Phi}\right)$, result in

$$
\boldsymbol{q}=\left(\boldsymbol{C}_{0} \boldsymbol{\Phi}\right)^{-1} \boldsymbol{y} .
$$

A first order digital ButTERWORTH highpass filter is used to approximate the modal velocities $\dot{\boldsymbol{q}}$ and a discrete integrator sums up the modal displacements $\boldsymbol{q}$ to get the integral state vector (15). The integration (15) starts with the beginning of active buckling control at $t_{\mathrm{LPV}}$ and continues indefinitely. The state vector $\boldsymbol{x}$ is used to calculate the control forces $\boldsymbol{u}$ via (19). The resulting $d S P A C E$ output voltages are filtered by analogue elliptic lowpass reconstruction filters before being amplified and applied to the piezoelectric stack actuators in piezo-elastic supports A and B. 


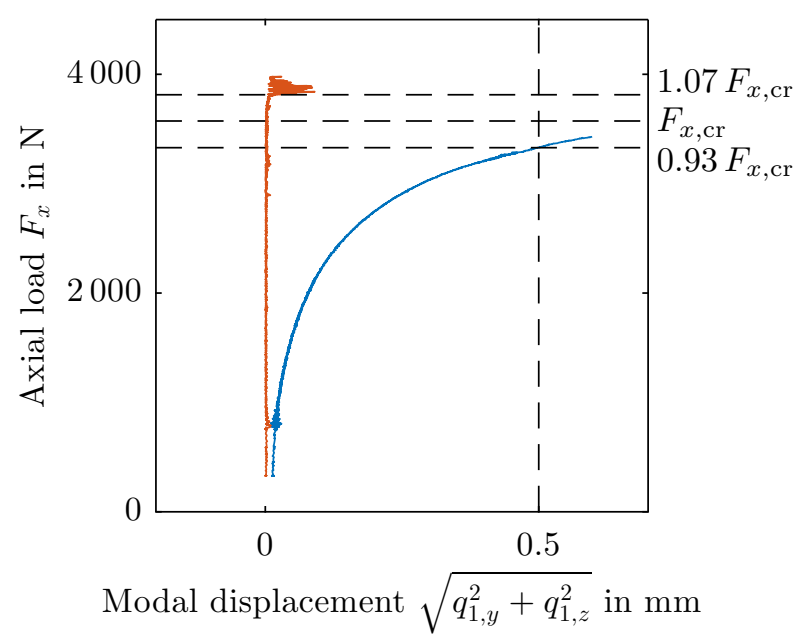

(a)

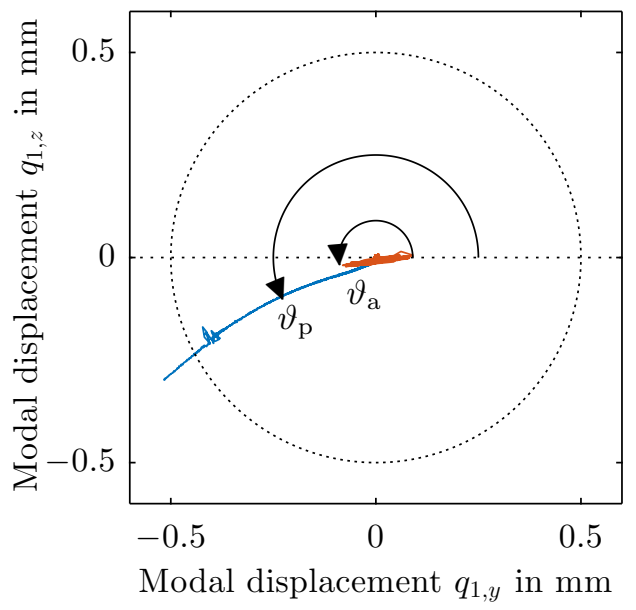

(b)

Fig. 5 Modal displacements $q_{1, y}$ and $q_{1, z}$ of the first modes in $y$ - and $z$-direction for the beam-column with $(-)$ and without (-) active buckling control, (a) absolute modal displacement versus axial load $F_{x}$, (b) modal displacements in $y$-z-plane

The results of the beam-column system with and without active buckling control are shown in figure 5 . The absolute modal displacements $q_{1, y}$ and $q_{1, z}$ of the first modes in $y$ - and $z$-direction are plotted with respect to the axial load $F_{x}$ in figure $5 \mathrm{a}$. Figure $5 \mathrm{~b}$ shows the modal displacements $q_{1, y}$ and $q_{1, z}$ in the $y$-z-plane.

Without active buckling control, the load-displacement curve shows the typical continuous deformation with increasing axial load which is known from real, imperfect beam-columns, [1]. Sudden buckling does not occur. In theory, the ideal beam-column with circular cross-section has infinite directions of buckling. The real beam-column, however, continuously deforms in an angle of $\vartheta_{\mathrm{p}}=202^{\circ}$ measured from the positive $y$-direction. The direction of buckling of the real uncontrolled system is mainly dependent on the predeflection of the beam-column and the assembly conditions of the test setup and varies accordingly. It is not possible to detect one single critical buckling load of the uncontrolled system, only a maximum admissible deformation can be set depending on the intended application. Here, a maximum admissible deformation of $0.5 \mathrm{~mm}$ is defined to avoid plastic deformation of the beam-column. It is reached for an axial load of $F_{x, 0.5}=3329 \mathrm{~N}=0.93 F_{x, \text { cr }}$ which is less than the theoretical critical buckling load $F_{x, \mathrm{cr}}=3573 \mathrm{~N}$ determined from the $\mathrm{FE}$ model via $(9)$.

With active buckling control, the piezoelectric stack actuators in the piezo-elastic supports are able to initially force the beam-column into a straight position and then to reduce the beam-column deformation for the ramp-shaped axial load $F_{x}$. The LPV control is able to stabilize the beam-column up to an axial load of $F_{x, \max }=3816 \mathrm{~N}=$ $1.07 F_{x, \mathrm{cr}}=1.14 F_{x, 0.5}$. Up to $F_{x, \max }$, the deformation in both $y$ - and $z$-direction remains zero, figure $5 \mathrm{a}$. At $F_{x, \max }$, the beam-column starts to oscillate and finally buckles at an angle of $\vartheta_{\mathrm{a}}=194^{\circ}$ in approximately the same direction as the passive beam-column, figure $5 \mathrm{~b}$.

\section{CONCLUSION}

Active Linear Parametric Varying (LPV) buckling control of an axially loaded beam-column with circular cross-section embedded in piezo-elastic supports is investigated experimentally. With the piezo-elastic supports, lateral forces of piezoelectric stack actuators are transformed into bending moments acting in arbitrary directions at the beam-column's ends. A modal model of the beam-column system based on a finite element (FE) model that is validated by an experimental system identification. The modal model is augmented by integrated states and an LPV control is implemented. The experiments show that the axially loaded beam-column with circular cross-section without active buckling control exhibits high lateral deformations for axial loads considerably below the theoretical critical buckling load. With active buckling control using the presented piezo-elastic supports, stabilization of the beam-column in arbitrary direction is possible. The LPV control is able to stabilize the beam-column for axial loads $14 \%$ higher than 
the passive beam-column and above the theoretical critical buckling load. In future investigations, active buckling control will be applied for step-shaped axial compressive loads where active buckling control is assumed to be very beneficial. Furthermore, system identification for higher axial loads may improve the controller model to avoid instability at higher axial loads.

\section{ACKNOWLEDGMENTS}

The authors like to thank the German Research Foundation (DFG) for funding this project within the Collaborative Research Center (SFB) 805.

\section{REFERENCES}

[1] S. P. Timoshenko and J. M. Gere. Theory of Elastic Stability. McGraw-Hill, New York, 1961.

[2] T. Meressi and B. Paden. Buckling control of a flexible beam using piezoelectric actuators. Journal of Guidance, Control, and Dynamics, 16(5):977-980, 1993.

[3] Q. S. Wang. Active buckling control of beams using piezoelectric actuators and strain gauge sensors. Smart Materials and Structures, 19:1-8, 2010.

[4] S. P. Thompson and J. Loughlan. The active buckling control of some composite column strips using piezoceramic actuators. Composite Structures, 32:59-67, 1995.

[5] A. A. Berlin, J. G. Chase, M. Yim, J. B. Maclean, M. Olivier, and S. C. Jacobsen. Mems-based control of structural dynamic instability. Journal of Intelligent Material Systems and Structures, 9:574- 586, 1998.

[6] G. Zenz and A. Humer. Stability enhancement of beam-type structures by piezoelectric transducers: theoretical, numerical and experimental investigations. Acta Mechanica, 226(12):3961-3976, 2015.

[7] G. C. Enss, R. Platz, and H. Hanselka. Uncertainty in loading and control of an active column critical to buckling. Shock and Vibration, 19:929-937, 2012.

[8] G. C. Enss and R. Platz. Evaluation of uncertainty in experimental active buckling control of a slender beam-column using weibull analysis. Mechanical Systems and Signal Processing, 79:123-131, 2016.

[9] M. Schaeffner, B. Götz, and R. Platz. Active buckling control of a beam-column with circular cross-section using piezoelastic supports and integral lqr control. Smart Materials and Structures, 25(6):065008, 2016.

[10] M. Schaeffner and R. Platz. Active buckling control of an imperfect beam-column with circular cross-section using piezoelastic supports and integral LQR control. Journal of Physics: Conference Series, 744:012165, 2016.

[11] G. C. Enss, C. M. Gehb, B. Goetz, T. Melz, S. Ondoua, Platz R., and M. Schaeffner. Device for bearing design elements in lightweight structures (Festkörperlager) Patent. DE 102015101084 A1, July 2016.

[12] J. S. Przemieniecki. Theory of Matrix Structural Analysis. McGraw-Hill, New York, 1968.

[13] W. Gawronski. Advanced Structural Dynamic and Active Control of Structures. Springer, 2010.

[14] E. Hendricks, O. Jannerup, and P. H. Sørensen. Linear Systems Control. Springer, 2008.

[15] A. Pandey, M. Sehr, and M. de Oliveira. Pre-filtering in gain-scheduled and robust control. In Proceedings of 2016 American Control Conference (ACC), Boston, MA, USA, July 6-8, 2016.

[16] J. Löfberg. Yalmip : A toolbox for modeling and optimization in MATLAB. In Proceedings of the CACSD Conference, Taipei, Taiwan, 2004. 